\title{
An extension of Ratner's rigidity theorem to n-dimensional hyperbolic space
}

\author{
LIVIO FLAMINIO \\ Department of Mathematics, University of Maryland, College Park, MD 20740, USA \\ and \\ Department of Mathematics, Stanford University, Stanford, CA 94305, USA
}

(Received 22 August 1985 and revised 25 February 1986)

Abstract. We prove that the horospherical foliations of two compact manifolds of constant negative curvature are measurably isomorphic if and only if the two manifolds are isometric.

\section{0 . Introduction}

The aim of this note is to give an extension of M. Ratner's theorem [Ra] on the rigidity of horocycle flows.

M. Ratner's theorem says that if $\Gamma_{1}$ and $\Gamma_{2}$ are two co-compact (more generally co-finite volume) discrete subgroups of $\mathrm{SL}_{2}(\mathbb{R})$ and $\psi: \Gamma_{1} \backslash \mathrm{SL}_{2}(\mathbb{R}) \rightarrow \Gamma_{2} \backslash \mathrm{SL}_{2}(\mathbb{R})$ is a bi-measurable map which preserves the Haar-measure and is equivariant for the action on the right of the horocycle group then there exists a $g \in \mathrm{SL}_{2}(\mathbb{R})$ such that

$$
\bar{\psi}\left(\Gamma_{1} x\right)=g \Gamma_{1} x h=\Gamma_{2} g x h,
$$

where $\bar{\psi}$ coincides a.e. with $\psi$ and $h$ is an element of the horocycle group. One can replace $\mathrm{SL}_{2}(\mathbb{R})$ with $\mathrm{PSL}_{2}(\mathbb{R}) \sim \mathrm{SO}_{0}(1,2)$ in which case the equivalent geometrical formulation of the theorem states that if $M_{1}$ and $M_{2}$ are two oriented surfaces of constant negative curvature -1 and $\psi: T^{1} M_{1} \rightarrow T^{1} M_{2}$ is a measure theoretical isomorphism of the horocycle flow (here $T^{1} M_{i}$ indicates the unit tangent bundle to $\boldsymbol{M}_{i}$ ) then $\psi$, up to a constant translation along the flow, is the lift to unit tangent bundles of an isometry $\tilde{\psi}: M_{1} \rightarrow M_{2}$.

In this paper we will prove the following generalization of Ratner's theorem:

THEOREM 1. Let $n$ be an integer $>2, \Gamma_{1}, \Gamma_{2}$ discrete co-compact subgroups of isometries of $\mathbb{H}^{n}$ - the hyperbolic n-dimensional space - and define $M_{i}={ }^{\text {def }} \Gamma_{i} \backslash \mathbb{\mathbb { H } ^ { n }}$; let $\psi: T^{1} M_{1} \rightarrow$ $T^{1} M_{2}$ be a bi-measurable isomorphism of the expanding horospherical foliation; then $\psi$ is the differential of an isometry $\tilde{\psi}: M_{1} \rightarrow M_{2}$.

We shall specify later exactly what we mean by bi-measurable isomorphism of the expanding horospherical foliation.

The proof of the above theorem exploits the main ideas of Ratner's paper [Ra], that is the technique of bootstrapping, via the 'polynomial rigidity' of horospheres, from expanding horospheres to geodesics and then to contracting horospheres.

\section{Notations and preliminaries}

(1.1) Hyperbolic space. For the sake of completeness, and for notational purposes as well, we shall start by recalling well known facts about hyperbolic space. 
We shall write $\mathrm{SO}_{0}(1, n)$ for the connected component of the identity of the group of $(n+1)$ by $(n+1)$ real matrices preserving the bilinear form

$$
\langle\mathbf{x}, \mathbf{y}\rangle=x_{0} y_{0}-\sum_{i=1}^{n} x_{i} y_{i}
$$

$\mathrm{SO}_{0}(1, n)$ acts on $\mathbb{R}^{n+1}$ in the standard way and the orbit of the point $\mathscr{I}={ }^{\text {def }}$ $(1,0, \ldots, 0)$ consists of the sheet of hyperboloid

$$
\Sigma \stackrel{\text { def }}{=}\left\{\mathbf{x} \in \mathbb{R}^{n+1} \mid x_{0}>0,\langle\mathbf{x}, \mathbf{x}\rangle=1\right\} ;
$$

$\mathrm{SO}_{0}(1, n)$ acts transitively and effectively on $\Sigma$, and the restriction of $-\langle\cdot, \cdot\rangle$ to the tangent bundle of $\Sigma$ defines a positive definite metric on $\Sigma$, which is of course invariant under the action of $\mathrm{SO}_{0}(1, n)$.

The stability group of $\mathscr{I}$ is given by the matrices of the type

$$
\left(\begin{array}{c|ccc}
1 & 0 & \cdots & 0 \\
\hline 0 & & & \\
\vdots & & K &
\end{array}\right) \quad K \in \operatorname{SO}(n) .
$$

Therefore there is an identification

$$
\Sigma \sim \mathrm{SO}_{0}(1, n) / \mathrm{SO}(n)
$$

which associates at every $p \in \Sigma$ the cosets of matrices $g$ SO $(n)$ where $g$ is some matrix for which $g(\mathscr{I})=p$.

The action of SO $(n)$ on the two dimensional planes tangent at $\mathscr{I}$ is transitive and therefore $\Sigma$ has a constant (sectional) curvature; it can be computed (see for example [Ko-No]) that the curvature equals -1 . $\Sigma$ endowed with this metric will be denoted by $\mathbb{H}^{n}$. It is well known [Ko-No] that all complete simply connected spaces of the same dimension whose curvature is a given constant are isometric, and therefore we are authorized to call $\mathbb{H}^{n}$ the space of negative curvature -1 .

(1.2) The geodesic flow. The metric of $\mathbb{H}^{n}$ determines the geodesic flow on $T^{1} \mathbb{H}^{n}$ :

$$
g:(v, t) \in T^{1} \mathbb{H}^{n} \times \mathbb{R} \mapsto g, v \in T^{1} \mathbb{H}^{n} .
$$

$T^{1} \mathbb{H}^{n}$ can be identified with the homogeneous space $\mathrm{SO}_{0}(1, n) / \mathrm{SO}(n-1)$, where $\mathrm{SO}(n-1)$ embeds in $\mathrm{SO}_{0}(1, n)$ by

$$
K \in \mathrm{SO}(n-1) \hookrightarrow\left(\begin{array}{cc|ccc}
1 & 0 & 0 & \cdots & 0 \\
0 & 1 & 0 & \cdots & 0 \\
\hline 0 & & & & \\
\vdots & \vdots & & K & \\
0 & 0 & &
\end{array}\right)
$$

In fact the above copy of $\mathrm{SO}(n-1)$ in $\mathrm{SO}_{0}(1, n)$ is immediately seen to be the group of isometries of $\mathbb{H}^{n}$ fixing the vector $\varepsilon=\operatorname{def}(0,1,0, \ldots, 0)$ tangent at the point $I$; then, for every $v$ tangent vector to $\mathbb{H}^{n}$, there is a unique coset $g \mathrm{SO}(n-1)$ of elements in $\mathrm{SO}_{0}(1, n)$ sending $\varepsilon$ to $v$. 
With this identification, the geodesic flow is easily seen to be given by the projection to $\mathrm{SO}_{0}(1, n) / \mathrm{SO}(n-1)$ of the right action on $\mathrm{SO}_{0}(1, n)$ of the one parameter subgroup of $\mathrm{SO}_{0}(1, n)$ given by

$$
G_{t} \stackrel{\text { def }}{=}\left(\begin{array}{cc|ccc}
\cosh t & \sinh t & 0 & \cdots & 0 \\
\sinh t & \cosh t & 0 & \ldots & 0 \\
\hline 0 & 0 & & & \\
\vdots & \vdots & & \operatorname{Id}_{n-1} & \\
0 & 0 & & &
\end{array}\right) \quad \text { where } t \in \mathbb{R}
$$

The fact that the right action of this group, which we will call henceforth the geodesic group, projects from $\mathrm{SO}_{0}(1, n)$ down to $T^{1} \mathbb{H}^{n}$ is a consequence of the commutation rules:

$$
G_{i} K=K G_{i} \quad \forall K \in \mathrm{SO}(n-1) \text { and } \forall G_{i} .
$$

Multiplication on the right by the one-parameter subgroup $\left(G_{t}\right)_{t \in \mathbb{R}}$ defines a flow on $\mathrm{SO}_{0}(1, n)$ which, with abuse of language, we shall call the geodesic flow on $\mathrm{SO}_{0}(1, n)$.

(1.3) The orthogonal frame bundle. We will indicate with $\mathrm{pr}, \mathrm{pr}_{1}$ the projection maps

$$
\begin{gathered}
\operatorname{pr}: \mathrm{SO}_{0}(1, n) \rightarrow \mathrm{SO}_{0}(1, n) / \mathrm{SO}(n) \sim \mathbb{H}^{n} \\
\mathrm{pr}_{1}: \mathrm{SO}_{0}(1, n) \rightarrow \mathrm{SO}_{0}(1, n) / \mathrm{SO}(n-1) \sim T^{1} \mathbb{H}^{n} ;
\end{gathered}
$$

they are given in the coordinates that we have chosen for $\mathbb{H}^{n}$ and $T^{1} H^{n}$ by

$$
g=\left(g_{i j}\right)_{i, j=0}^{n} \mapsto \operatorname{pr}(g)=\left(\begin{array}{c}
g_{00} \\
\vdots \\
g_{n 0}
\end{array}\right)
$$

and

$$
g=\left(g_{i j}\right)_{i, j=0}^{n} \mapsto \operatorname{pr}_{1}(t)=\left(\begin{array}{cc}
g_{00} & g_{01} \\
\vdots & \vdots \\
g_{n 0} & g_{n 1}
\end{array}\right) ;
$$

$\mathrm{SO}_{0}(1, n)$ itself can be considered as the orthogonal frame bundle to $T^{1} \mathbb{H} \mathbb{H}^{n}$, which we will indicate with $F H^{n}$; in fact for every matrix $g \in \mathrm{SO}_{0}(1, n)$, the first column gives the coordinates of a point $p \in \mathbb{H}^{n}$ and the remaining $n$ columns the coordinates of $n$ orthogonal vectors tangent to $\mathbb{H}^{n}$ at $p$. Thus we have dual languages

$$
\begin{aligned}
& \frac{\text { algebraic }}{\mathrm{SO}_{0}(1, n)} \quad \frac{\text { geometrical }}{F H^{n}} \\
& \mathrm{SO}_{0}(1, n) / \mathrm{SO}(n-1) \quad T^{1} \mathbb{H}^{n} \\
& \mathrm{SO}_{0}(1, n) / \mathrm{SO}(n) \quad \mathbb{O}^{n} \text {. }
\end{aligned}
$$

(1.4) The horospherical group. The subgroup of $\mathrm{SO}_{0}(1, n)$ that interests us the most is the '(expanding) horospherical group'; it can be defined as the group whose elements leave fixed the (projective) point at $-\infty$ of the geodesic issued from $\varepsilon$ and have no other fixed point on the boundary of $\mathbb{H}^{n}$. The geodesic issued from $\varepsilon$ is given in our coordinates by the map

$$
t \in \mathbb{R} \mapsto(\cosh t, \sinh t, 0, \ldots, 0),
$$


and therefore its projective point at $-\infty$ is the point $(1,-1,0, \ldots, 0)$; the Lie-algebra of the expanding horospherical group must have this vector as an eigenvector and therefore its elements have the form:

$$
\left(\begin{array}{cc|rrr}
a & 0 & a_{2} & \cdots & a_{n} \\
0 & a & -a_{2} & \cdots & -a_{n} \\
\hline a_{2} & a_{2} & & & \\
\vdots & \vdots & & K & \\
a_{n} & a_{n} & & &
\end{array}\right) \quad K \in \operatorname{SO}(n-1)
$$

But elements of the type

$$
\left(\begin{array}{cc|ccc}
a & 0 & 0 & \cdots & 0 \\
0 & a & 0 & \cdots & 0 \\
\hline 0 & 0 & & & \\
\vdots & \vdots & & K &
\end{array}\right) \quad K \in \operatorname{SO}(n-1)
$$

also leave invariant the point $(1,1,0 \ldots, 0)$ and, by our definition must, be excluded from the horospherical Lie-algebra; one is then left with elements

$$
\left(\begin{array}{cc|ccc}
0 & 0 & a_{2} & \cdots & a_{n} \\
0 & 0 & -a_{2} & \cdots & -a_{n} \\
\hline a_{2} & a_{2} & & & \\
\vdots & \vdots & & 0 & \\
a_{n} & a_{n} & &
\end{array}\right)
$$

which generate the group of matrices

$$
n_{\mathbf{a}} \stackrel{\operatorname{def}}{=}\left(\begin{array}{cc|ccc}
1+\|\mathbf{a}\| / 2 & \|\mathbf{a}\| / 2 & a_{2} & \cdots & a_{n} \\
-\|\mathbf{a}\| / 2 & 1-\|\mathbf{a}\| / 2 & -a_{2} & \cdots & -a_{n} \\
\hline a_{2} & a_{2} & & \\
\vdots & \vdots & \operatorname{Id}_{n-1} &
\end{array}\right),
$$

where we have set $\mathbf{a}=\left(a_{2}, \ldots, a_{n}\right)$ and $\|\mathbf{a}\|={ }^{\text {def }} \sqrt{\sum_{2}^{n} a_{i}^{2}}$. Notice that

$$
n_{\mathrm{a}} n_{\mathrm{b}}=n_{\mathrm{a}+\mathrm{b}}
$$

and therefore the horospherical group is isomorphic to the additive group $\mathbb{R}^{n-1}$. Its one-parameter subgroups are therefore given by

$$
n_{s \mathbf{a}} \quad s \in \mathbb{R},
$$

where a is some element in $\mathbb{R}^{n-1}$. We will denote the expanding horospherical group with the letter $\mathcal{N}$.

Similarly one can define the contracting horospherical group $\mathcal{M}$ as the sub-group of isometries which have, as their unique fixed point, the point at $+\infty$ of the geodesic issued from $\varepsilon$ to the additive group $\mathbb{R}^{n}$ and one can easily verify that $\mathcal{M}$ is given 
by the matrices

$$
m_{\mathbf{a}} \stackrel{\operatorname{def}}{=}\left(\begin{array}{cc|ccc}
1+\|\mathbf{a}\| / 2 & -\|\mathbf{a}\| / 2 & a_{2} & \cdots & a_{n} \\
\|\mathbf{a}\| / 2 & 1-\|\mathbf{a}\| / 2 & a_{2} & \cdots & a_{n} \\
\hline a_{2} & -a_{2} & & & \\
\vdots & \vdots & & \operatorname{Id}_{n-1} & \\
a_{n} & -a_{n} & &
\end{array}\right),
$$

where $\mathbf{a}=\left(a_{2}, \ldots, a_{n}\right)$ is some element in $\mathbb{R}^{\mathrm{n}-1}$. One can also see that $\mathcal{M}$ is isomorphic to $\mathbb{R}^{n-1}$ because

$$
m_{\mathrm{a}} m_{\mathrm{b}}=m_{\mathbf{a}+\mathbf{b}}
$$

The reason for calling these groups expanding and contracting lies in their commutation properties with the geodesic flow:

$$
\begin{array}{rlrl}
n_{\mathbf{a}} G_{t} & =G_{t} n_{e^{1}} & \forall n_{\mathbf{a}} \in \mathcal{N}, \\
m_{\mathbf{a}} G_{t}=G_{t} m_{e^{-t}} & \forall m_{\mathbf{a}} \in \mathcal{M} .
\end{array}
$$

Also it is important for us to notice the commutation relations of the horospherical groups with the group $\mathrm{SO}(n-1)$ :

$$
\begin{array}{ll}
n_{\mathrm{a}} K=K n_{\mathrm{a} K} & \forall n_{\mathrm{a}} \in \mathcal{N}, \forall K \in \mathrm{SO}(n-1), \\
m_{\mathrm{a}} K=K m_{\mathrm{a} K} & \forall m_{\mathrm{a}} \in \mathcal{M}, \forall K \in \mathrm{SO}(n-1),
\end{array}
$$

where by $\mathbf{a} K$ it is meant the ordinary matrix multiplication on the right; the orbit under $\mathcal{N}(M)$ of a point $g \in \mathrm{SO}_{0}(1, n)$ is called the expanding (contracting) horosphere through $g$.

(1.5) Horospheres in $T^{1} \mathbb{H}^{n}$. The relations (*) and (**) show that, unlike the action of the geodesic group, the actions of the horospherical groups do not project from $\mathrm{SO}_{0}(1, n)$ to $\mathrm{SO}_{0}(1, n) / \mathrm{SO}(n-1) \sim T^{1} \mathbb{W}^{n}$; nevertheless the same relations also show that if two orbits of the horospherical group have projections in $T^{1} \mathbb{H}^{n}$ that intersect at a point then these projections are identical: in fact, in the case of $\mathcal{N}$, one has that if $\operatorname{pr}_{1}\left(g n_{\mathrm{a}}\right)=\operatorname{pr}_{1}\left(g^{\prime} n_{\mathrm{b}}\right)$ for some $\mathrm{a}$ and $\mathrm{b}$ then $g n_{\mathrm{a}}=g^{\prime} n_{\mathrm{b}} K$ and therefore

$$
\begin{aligned}
& g n_{\mathrm{c}}=g^{\prime} n_{\mathbf{b}} K n_{\mathrm{c}-\mathbf{a}}=g^{\prime} n_{\mathbf{b}+(\mathbf{c}-\mathbf{a}) K^{-1} K} \\
\Rightarrow & \operatorname{pr}_{1}\left(g n_{\mathrm{c}}\right)=\operatorname{pr}_{1}\left(g^{\prime} n_{\left.\mathbf{b}+(\mathbf{c}-\mathbf{a}) K^{-1} K\right)}\right. \\
\Rightarrow & \left(\mathrm{pr}_{1}\left(g n_{\mathbf{c}}\right)\right)_{\mathbf{c} \in \mathbf{R}^{n-1}}=\left(\operatorname{pr}_{1}\left(g^{\prime} n_{\mathbf{c}}\right)\right)_{\mathbf{c} \in \mathbf{R}^{n-1}} .
\end{aligned}
$$

Similar computations hold for $\mathcal{M}$. Then we have that the projections of the expanding (contracting) horospheres in $\mathrm{SO}_{0}(1, n)$ are the leaves of a foliation in $T^{1} \mathrm{PD}^{n}$; such a foliation is called the expanding (contracting) horospherical foliation of $T^{1} \mathrm{H}^{n}$; in the language of Anosov systems this foliation is also called the unstable (stable) foliation determined by the geodesic flow. The leaves of the horospherical foliation of $T^{1} \mathbb{H}^{n}$ will be called horospheres, and this may create some confusion since we used the same name for the orbits of the horospherical group acting on $\mathrm{SO}_{0}(1, n)$. But whenever confusion may arise we shall specify which space we are considering; this will avoid having to expand the vocabulary unnecessarily.

It is also important for us to notice that given a point $v \in T^{1} \mathbb{H}^{n}$ and a $g \in \mathrm{SO}_{0}(1, n)$ that projects to $v$, for every other point $w$ belonging to the expanding horosphere 
of $v$ one can find a unique horospherical element $n_{\mathrm{a}}$ such that

$$
\operatorname{pr}_{1}\left(g n_{\mathbf{a}}\right)=w ;
$$

in other words the restriction of the projection map $\mathrm{pr}_{1}$ to a expanding horosphere in $\mathrm{SO}_{0}(1, n)$ is a one-to-one map onto a horosphere in $T^{1} \mathbb{H}^{n}$; in fact if

then one has

$$
\operatorname{pr}_{1}\left(g n_{\mathbf{a}}\right)=w=\operatorname{pr}_{1}\left(g n_{\mathrm{b}}\right)
$$

$$
g n_{\mathrm{a}}=g n_{\mathrm{b}} K, \quad \text { for some } K \in \mathrm{SO}(n-1)
$$

which implies $n_{\mathrm{a}}=n_{\mathrm{b}}$. A similar statement is true for contracting horospheres.

We will consider on $T^{1} \mathbb{H}^{n}$ a metric invariant under the left action of $\mathrm{O}(1, n)$. For every such metric, contracting horospheres, expanding horospheres and geodesics are mutually orthogonal; therefore the metric is uniquely defined by assigning the length of a vector tangent to a horosphere and of a vector tangent to a geodesic. The simplest possible choice is that in which:

(a) geodesics in $T^{1} \mathbb{T}^{n}$ have the same length as their projections in $\mathbb{H}^{n}$

(b) points $u$ and $v$ on an expanding (contracting) horosphere in $T^{1} \mathbb{H}^{n}$ have distance equal to $\|\mathbf{a}\|$ where $n_{\mathrm{a}}\left(m_{\mathrm{a}}\right)$, with $\mathbf{a} \in \mathbb{R}^{n-1}$, is a horospherical element that maps $u$ to $v$. We will denote the distance induced by this metric by $d(\cdot, \cdot)$.

Other foliations that we will consider are the weakly unstable (weakly stable) foliations. Let $\mathcal{O}$ be an expanding (contracting) horosphere; then a leaf of the weakly unstable (stable) foliation is given by $\bigcup_{t \in R} g_{t} \mathcal{O}$. It can be seen that two points $x, y$ in $T^{1} H^{n}$ belong to the same weakly unstable (stable) leaf if and only if $d\left(g_{t} x, g_{t} y\right)$ is bounded for all $t>0$ (for all $t<0$ ).

(1.6) Horocycles and horolines

Definition. An expanding horocycle in $\mathrm{SO}_{0}(1, n)$ is an orbit of some one-parameter subgroup of $\mathcal{N}$ :

$$
t \in \mathbb{R} \mapsto g n_{s a},
$$

where $g$ is some point in $\operatorname{SO}_{0}(1, n)$ and $\mathbf{a} \in \mathbb{R}^{n}$ is normalized so that $\|\mathbf{a}\|=1$.

An arc of a horocycle is a finite segment of the orbit of such a subgroup. Contracting horocycles are defined similarly.

Definition. A horoline (an arc of a horoline) in $T^{1} \mathbb{H}^{n}$ is the projection to $T^{1} \mathbb{H}^{n}$ of a horocycle (of an arc of horocycle).

Note that horolines are well defined; in other words if one considers a horocyle on some horosphere in $\mathrm{SO}_{0}(1, n)$, projects it down to $T^{1} \mathrm{H}^{n}$ and finally lifts it back to a different covering horosphere in $\mathrm{SO}_{0}(1, n)$, the curve one obtains is still a horocycle.

Definition. Two horolines (arcs of horoline) are parallel if they are the projection to $T^{1} \mathbb{H}^{n}$ of horocycles in $\mathrm{SO}_{0}(1, n)$ given by the action of the same one-parameter subgroup of $\mathcal{N}$ on points on the same horosphere in $\mathrm{SO}_{0}(1, n)$.

Once again, parallelicity is well defined because it is independent of the lift that one considers. Note that in particular parallel arcs of horolines lie in the same horosphere. To denote a horoline we shall use the notation

$$
\left(u_{s}\right)_{s \in I} \quad \text { for } I \text { an interval in } \mathbb{R} \text { and } u_{s} \in T^{1} \mathbb{H}^{n}
$$


and with this it is meant that there exists a $g \in \mathrm{SO}_{0}(1, n)$ and a $n_{\mathrm{a}} \in \mathcal{N}$, with $\|\mathbf{a}\|=1$, such that

$$
u_{s}=\operatorname{pr}_{1}\left(g n_{s \mathbf{a}}\right) \quad \forall s \in I
$$

It is interesting to notice that horolines are geodesic lines on horospheres in $T^{1} \mathbb{H}^{n}$ with respect to the induced metric.

LEMMA. Let $\left(u_{s}\right)_{0}^{\infty},\left(v_{s}\right)_{0}^{\infty}$ be two arcs of horolines in $T^{1} \mathbb{H}^{n}$ such that

$$
\sup _{0<s<\infty} d\left(u_{s}, v_{s}\right)<+\infty
$$

Then $\left(u_{s}\right)_{0}^{\infty}$ and $\left(v_{s}\right)_{0}^{\infty}$ belong to the same horosphere and are parallel; in particular this implies $d\left(u_{s}, v_{s}\right)=$ constant.

The proof is immediate: by the hypothesis the two horolines have the same point at $+\infty$ and therefore belong to the same weakly unstable leaf; this easily implies that they are actually on the same horosphere. On the other hand the geometry of a horosphere with the metric we are considering is euclidean and the distance of two horolines on a horosphere is bounded if and only if their distance is bounded in the metric of the horosphere; and this ends the proof.

(1.7) Compact quotients. Now let $\Gamma$ be a discrete co-compact group of isometries of $\mathbb{H}^{n}$, i.e. a discrete subgroup of $\mathrm{SO}_{0}(1, n)$ such that $\Gamma \backslash \mathbb{H}^{n}$ is compact; to keep the notation short we write $M$ for the $n$-dimensional manifold $\Gamma \backslash \mathbb{H}^{n}$ and we will call manifolds obtained in this way compact quotients of $\mathbb{H}^{n}$. Then $\Gamma \backslash \mathrm{SO}_{0}(1, n) /$ SO $(n-1)$ is the unit tangent bundle $T^{1} M$ of $M$, and $\Gamma \backslash S_{0}(1, n)$ is the orthonormal frame bundle $F M$ of $M$. Since $\Gamma$ acts on $H^{n}$ on the left, the action of the geodesic group on $T^{1} M$ and $F M$ still makes sense, and so does the action of the horospherical group and its one-parameter groups on $F M$; if $f$ is a frame of $F M$ we will denote the action of the geodesic group, of the horospherical groups, and of SO $(n-1)$, by

$$
g_{t}(f), \quad n_{\mathbf{a}}(f), \quad m_{\mathbf{a}}(f), \quad K(f) .
$$

On $T^{1} M$ we can still consider the horospherical foliation whose leaves are the projection from $F M$ to $T^{1} M$ of the orbits under the horospherical group of frames of $F M$; similarly one defines horolines and parallel horolines in $T^{1} M$ as we did for $T^{1} \mathbb{H}^{n}$. Notice also that since the metric on $T^{1} \mathbb{H}^{n}$ that we are considering is left invariant, it also determines a metric on $T^{1} M$ which has the property that arcs of geodesics and horolines have lengths equal to their parametrization.

Now, if $M_{1}$ and $M_{2}$ are two compact quotients of $\mathbb{H}^{n}$, it is clear what we mean for a bi-measurable map $\psi: T^{1} M_{1} \rightarrow T^{1} M_{2}$ to be an isomorphism of the expanding horospherical foliation: we mean that $\psi$ maps almost every expanding horosphere in $T^{1} M_{1}$ to an expanding horosphere preserving the induced metric.

(1.8) Measure on $T^{1} M$ and $F M$. The geodesic flow has a canonical smooth measure associated with it, the so-called Liouville measure. In the case that we are considering, this measure also has a group theoretical interpretation. In fact $F H^{n} \sim$ $\mathrm{SO}_{0}(1, n)$ is a unimodular group, that is to say, has a measure invariant under both left and right translations; such a measure, by pullback of the projection map from 
$\mathrm{SO}_{0}(1, n)$ to $\mathrm{SO}_{0}(1, n) / G^{\prime}$, determines a measure on every coset space $\mathrm{SO}_{0}(1, n) / G^{\prime}$ (here $G^{\prime}$ stands for any subgroup of $\mathrm{SO}_{0}(1, n)$ ) which will be invariant under the left-action on $\mathrm{SO}_{0}(1, n) / G^{\prime}$ of $\mathrm{SO}_{0}(1, n)$ and by the right-action on $\mathrm{SO}_{0}(1, n) / G^{\prime}$ of those elements in $\mathrm{SO}_{0}(1, n)$ that commute with $G^{\prime}$. In particular in this way we can construct a measure on $T^{1} \mathbb{H}^{n}$ which will be invariant under the action of $\mathrm{SO}_{0}(1, n)$ and will also be preserved by the geodesic flow: such a measure coincides with the Liouville measure. Now let $\Gamma$ be a discrete group of isometries of $\mathbb{H}^{n}$ and $M$ the smooth manifold $\Gamma \backslash \mathbb{H}^{n}$; the left invariance of the Liouville measure on $T^{1} \mathfrak{H} \mathbb{Q}^{n}$ allows us to project it on the quotient space $\Gamma \backslash T^{1} \mathbb{H}^{n} \sim T^{1} M$ to obtain the Liouville measure on $T^{1} M$; henceforth when we will talk about the measure of any compact quotient of $T^{1} \mathbb{H}^{n}$ we will refer to the above measure normalized to have total mass 1. It is well known [Mo] that the Haar measure on compact quotients of $\mathrm{SO}_{0}(1, n)$ is an ergodic measure for the action of any element of the horospherical group; in particular this implies that the action of the full (expanding) horospherical group on compact quotients of $\mathrm{SO}_{0}(1, n)$ is ergodic which in turn implies that the horospherical foliation of a compact quotient of $T^{1} \mathbb{H}^{n}$ is ergodic.

We also will use the fact that the geodesic flow on any compact quotient of $\mathrm{SO}_{0}(1, n) / \mathrm{SO}(n-1)$ and $\mathrm{SO}_{0}(1, n)$ is ergodic which is again a consequence of Moore's theorem [Mo].

(1.9) Sets of full measure in $T^{1} M$. Here we present an argument that we will consider often in the sequel. Let $\Lambda$ be some subset in $T^{1} M$ and $\tilde{\Lambda}=\operatorname{pr}_{1}^{-1}(\Lambda)$ its lift to the frame bundle of $M$. Given a horospherical element $n_{\mathrm{a}}$ we have that the set of frames $f$ for which

$$
\frac{1}{t} \int_{0}^{t} \chi_{\tilde{\Lambda}}\left(n_{s \mathbf{a}}(f)\right) d s \rightarrow \tilde{\mu}(\tilde{\Lambda})=\mu(\Lambda)
$$

is a set $\tilde{\Lambda}_{\text {typ }}$ of full measure; therefore the set of $v \in T^{1} M$ for which $\left.\operatorname{pr}_{1}(\{v\}) \cap \tilde{\Lambda}_{\text {typ }}\right)$ is a set of full measure in $\operatorname{pr}_{1}(\{v\})$ is also a set of full measure in $T^{1} M$. This amounts to saying that, for any $\Lambda \subset T^{1} M$, the set of $v \in T^{1} M$ having the property that almost every horoline through $v$ visits the set $\Lambda$ with frequency eventually equal to $\mu(\Lambda)$ is a set of full measure in $T^{1} M$.

\section{A lemma on the average distance of horolines}

LeMmA 2.1. Let $\left(u_{s}\right),\left(v_{s}\right)-s \in \mathbb{R}$ - be continuous curves in a metric space $(X, d)$ and suppose that for every interval $(\alpha, \beta) \subset \mathbb{R}$ in which $d\left(u_{s}, v_{s}\right) \leq R$ there exists $a$ polynomial $P_{d}$ of degree at most $d$ for which

$$
\gamma^{-1} P_{d}(s) \leq d\left(u_{s}, v_{s}\right) \leq \gamma P_{d}(s)
$$

for all $s \in(\alpha, \beta)$; then $\forall t>0$ either

$$
\frac{1}{t} \int_{0}^{t} d\left(u_{s}, v_{s}\right) d s>\frac{R}{\gamma^{2} C_{d}},
$$

$C_{d}$ being a constant depending only on $d-$ or

$$
d\left(u_{s}, v_{s}\right)<R \quad \forall s \in(0, t) .
$$


Proof. The set $A={ }^{\operatorname{def}}\left\{s \in(0, t) \mid d\left(u_{s}, v_{s}\right)<R\right\}$ is an open set and hence union of open intervals $\left(\alpha_{i}, \beta_{i}\right)$; on the complement of $A$ the average of $d\left(u_{s}, v_{s}\right)$ is certainly greater than $R / \gamma^{2} C_{d}$, so we need to worry only about the intervals $\left(\alpha_{i}, \beta_{i}\right)$. But on each such an interval the average of $d\left(u_{s}, v_{s}\right)$ is bounded from below by the average of a polynomial $\gamma^{-1} P_{d}(s)$ and the average of a polynomial is bounded from below by its sup norm divided by a constant $C_{d}$ which depends only upon its degree. But

$$
\sup _{\alpha_{i} \leq s \leq \beta_{i}} \frac{P_{d}(s)}{\gamma C_{d}} \geq \frac{1}{\gamma C_{d}} \max \left(P_{d}\left(\alpha_{i}\right), P_{d}\left(\beta_{i}\right)\right) \geq \frac{R}{\gamma^{2} C_{d}} .
$$

Therefore the average of $d\left(u_{s}, v_{s}\right)$ can be smaller than $R / \gamma^{2} C_{d}$ only if it never reaches $R$.

LEMMA 2.2. Let $\left(u_{s}\right),\left(v_{s}\right)$ be horolines in $T^{1} M_{2}$; there exists an $R$ smaller than the radius of injectivity of $T^{1} M_{2}$ such that if $d\left(u_{s}, v_{s}\right)<R, \forall s \in[\alpha, \beta]$, then in the same interval one has

$$
\frac{P(s)}{\gamma}<d\left(u_{s}, v_{s}\right)<\gamma P(s),
$$

where $P$ is some polynomial of degree at most 4 , and $\gamma$ some universal constant.

Proof. $d\left(u_{s}, v_{s}\right)$ being always less than $R, u_{s}$ and $v_{s}$ can be lifted to points $\bar{u}_{s}$ and $\bar{v}_{s}$ in $T^{1} \mathbb{H}^{n}$ so that $d\left(u_{s}, v_{s}\right)=d\left(\bar{u}_{s}, \bar{v}_{s}\right)$ for all $s \in[\alpha, \beta] .\left(\bar{u}_{s}\right),\left(\bar{v}_{s}\right)$ are projections to $T^{1} \mathbb{H}^{n}$ of the orbits of two points $g_{1}, g_{2} \in G$ under two one-parameter subgroups $n_{s \mathrm{a}}, n_{s \mathrm{~b}}$ of the horospherical group $\mathcal{N} \subset G$. By the invariance of $d$ under isometries of $\mathbb{H}^{n}$ the distance between $u_{s}$ and $v_{s}$ equals the distance of the projection of the identity of $G$ from the projection of $n_{-s \mathbf{a}} g_{1}^{-1} g_{2} n_{s b}$, and the entries of the latter matrix are polynomials of degree at most 4 in $s$. If $R$ is small enough one has that this distance is well approximated by the ordinary euclidean distance between the first two columns of the matrices id and $n_{-s \mathbf{a}} g_{1}^{-1} g_{2} n_{s b}$, which proves the statement.

LEMMA 2.3. Let $\left(u_{s}\right),\left(v_{s}\right)$ be horolines in $T^{1} M_{2}, D$ the diameter of $T^{1} M_{2}$ and $R, \gamma$ as in the above lemma. Suppose that on a set of density greater than $(1-\xi)$ of $s \in[0, t]$ one has $d\left(u_{s}, v_{s}\right)<\eta$. Then if $\xi<R / 2 \gamma^{2} D C_{4}$ and $\eta<R / 2 \gamma^{2} C_{4}$ one also has

$$
d\left(u_{s}, v_{s}\right)<R \quad \forall s \in[0, t] .
$$

Proof. From the hypotheses one has

$$
\frac{1}{t} \int_{0}^{t} d\left(u_{s}, v_{s}\right) d s<\eta+\xi D<\frac{R}{2 \gamma^{2} C_{4}}+\frac{R}{2 \gamma^{2} D C_{4}} D=\frac{R}{\gamma^{2} C_{4}} .
$$

Then the two previous lemmas imply the conclusion.

It should be pointed out that, although the above lemmas require the compactness of $M_{2}$, with a subtler argument (see [Ra]) rigidity can be proved for finite volume manifolds.

3. In which it is shown that the geodesic flow commutes with $\psi$

Henceforth $M_{1}$ and $M_{2}$ will indicate two compact quotients of $\mathbb{H}^{n}$; their unit tangent bundle will be endowed with a left invariant metric as in $\S 1.7$ and $\psi$ will be a 
bi-measurable map that maps isometrically almost every expanding horosphere to an expanding horosphere.

For every $\theta>0$ there exists a set $\Lambda_{\theta} \subset T^{1} M_{1}$ on which $\psi$ is uniformly continuous (cf. lemma 3.1 in [Ra]) and such that $\mu_{1} \Lambda_{\theta}>1-\theta$.

As we have shown in $\S 1.9$, by the ergodicity of the action of one-parameter subgroups of the horospherical group on $F M_{1}$, there exists a set $\bar{\Lambda}_{\theta} \subset T^{1} M_{1}$ of full $\mu_{1}$-measure such that $\forall x \in \bar{\Lambda}_{\theta}$ almost every horoline through $x$ intersects $\Lambda_{\theta}$ - the set of continuity of $\psi$ - with frequency eventually larger than $1-\theta$ : such horolines will be said to be 'typical' for $\Lambda_{\theta}$. Define

$\Omega \stackrel{\text { def }}{=}\left\{x \in T^{1} M_{1} \mid\right.$ almost every point of the horosphere of $x$ belongs to $\left.\bar{\Lambda}_{\theta}\right\}$.

$\Omega$ is a set of full measure in $T^{1} M_{1}$ entirely made of horospheres. Let us also introduce the notation $\pi_{x}$ to denote the horosphere to which the point $x$ belongs.

Proposition 3.1. For every $\delta>0$ there exists an $\eta>0$ such that if $|t|<\eta$ and $x, g_{t} x$ belong to $\Omega$, we have that:

(a) $g_{t}\left(\psi\left(\pi_{x}\right)\right)=\psi\left(\pi_{g_{t} x}\right)$;

(b) if $\left(u_{s}\right)$ is a horoline in $\pi_{x}$, then $g_{t}\left(\psi\left(u_{s}\right)\right)$ and $\psi g_{t}\left(u_{s}\right)$ are parallel horolines.

(c) $d\left(\psi g_{i} y, g_{i} \psi y\right)<2 \delta, \quad \forall y \in \pi_{x}$.

Proof. For all $\delta>0$ there exists an $\eta>0$ such that

$$
x, y \in \Lambda_{\theta} \text { and } d(x, y)<\eta \Rightarrow d(\psi x, \psi y)<\delta .
$$

We can assume $\eta<\delta$, and $t<\eta$. Since $x$ and $g_{t} x$ both belong to $\Omega$ there is a point $z \in \pi_{x}$ such that $z$ and $g_{t} z$ both belong to $\bar{\Lambda}_{\theta}$; thus we can then assume without loss of generality that $x$ and $g_{t} x$ actually belong to $\bar{\Lambda}_{\theta}$.

Let $\left(u_{s}\right)$ be a horoline through $x$ and consider the horolines

$$
w_{s} \stackrel{\text { def }}{=} g_{t}\left(\psi u_{e^{-t_{s}}}\right) \text { and } \quad v_{s} \stackrel{\text { def }}{=} \psi\left(g_{t} u_{e^{-t_{s}}}\right)
$$

one has

$$
\begin{aligned}
d\left(v_{s}, w_{s}\right) & \leq d\left(v_{s}, \psi u_{e^{-t_{s}}}\right)+d\left(\psi u_{e^{-t_{s}}}, w_{s}\right) \\
& \leq d\left(\psi\left(g_{t} u_{e^{-t_{s}}}\right), \psi\left(u_{e^{-t_{s}}}\right)\right)+t
\end{aligned}
$$

since the distance between $g_{t} u_{e^{-t_{s}}}$ and $u_{e} e_{s}$ is less than $\eta$, if both these points belong to $\Lambda_{\theta}$, one also has $d\left(\psi\left(g_{t} u_{e}^{-t_{s}}\right), \psi\left(u_{e^{-t_{s}}}\right)\right)<\delta$ and therefore

$$
d\left(u_{s}, w_{s}\right)<2 \delta .
$$

But almost every horoline $\left(u_{s}\right)$ through $x$ has the property that both $\left(u_{s}\right)$ and $\left(g_{t} u_{s}\right)$ are typical horolines for $\Lambda_{\theta}$, so both $g_{t} u_{e} e_{s}$ and $u_{e^{-t} s}$ will be in $\Lambda_{\theta}$ with frequency eventually larger than $1-2 \theta$. For such a horoline $\left(u_{s}\right)$, if $\theta$ and $\delta$ are chosen small enough, by lemma 2.3 one has that

$$
d\left(v_{s}, w_{s}\right)<R \quad \forall s \in \mathbb{R} .
$$

But then lemma 1.6 implies that $\left(v_{s}\right)$ and $\left(w_{s}\right)$ are parallel horolines; in particular they lie on the same horosphere; so we have proved that

$$
\pi_{\psi\left(g_{t} x\right)}=\psi\left(\pi_{g_{t} x}\right)
$$


In fact more is true: since we have that for a set of full measure of horolines $\left(\boldsymbol{u}_{s}\right)$, the corresponding horolines $\left(v_{s}\right)$ and $\left(w_{s}\right)$ are parallel, and since the map $\psi$ is assumed to be an isometry on horospheres, the same conclusion will actually hold for every horoline $\left(u_{s}\right)$ in $\pi_{x}$ and for a set of full measure of $x$, which is what (b) states. Finally, (c) follows from (a), (b), the fact that $\psi$ is an isometry on horospheres and the fact that it holds on a dense set of $y \in \pi_{x}$. The proof of proposition 3.1 is now complete.

Now let

$$
\Omega_{1} \stackrel{\text { def }}{=}\left\{x \in T^{1} M_{1} \mid \begin{array}{c}
\text { the weakly unstable leaf of } x \\
\text { intersects } \Omega \text { on a set of full measure }
\end{array}\right\} ;
$$

$\Omega_{1}$ is then the set of points whose geodesics visit $\Omega$ with frequency 1 and is a set of full measure completely made of weakly unstable leaves.

If $x \in \Omega \cap \Omega_{1}$ and we denote its weakly unstable leaf by $W(x)$, we have that $\psi$ maps $W(x) \cap \Omega$, which is dense in $W(x)$, to points in the weakly unstable leaf of $\psi(x)$; by proposition 3.1 this mapping is uniformly continuous on $W(x) \cap \Omega$ and therefore there is a unique continuous extension $\bar{\psi}$ of $\psi$ to the whole $W(x)$ that coincides a.e. with $\psi$ and maps the weakly unstable leaves of points in $\Omega_{1}$ to weakly unstable leaves. Thus we can assume without loss of generality that $\psi$ is continuous on the weakly unstable leaves in $\Omega_{1}$.

Now let $x \in \Omega_{1}$; by proposition 3.1 for every $t \in \mathbb{R}$ there is a unique point $\operatorname{Tr}[x ; t]$ on the horosphere of $x$ which has the following properties:

(1) $\psi\left(g_{t}(\operatorname{Tr}[x ; t])\right)=g_{t}(\psi(x))$;

(2) $d(x, \operatorname{Tr}[x ; t])<\delta(t)$ and $\lim _{t \rightarrow 0} \delta(t)=0$.

Our aim now is to show that for almost all $x$ and for all $t$, one has $\operatorname{Tr}[x ; t]=x$, in other words

$$
\psi\left(g_{i}(x)\right)=g_{i}(\psi(x)) .
$$

To this purpose let us first note that

$$
\operatorname{Tr}\left[x ; t+t^{\prime}\right]=g_{-t}\left(\operatorname{Tr}\left[g_{t}(\operatorname{Tr}[x ; t]) ; t^{\prime}\right]\right)
$$

and therefore the continuity in $t$ of $\operatorname{Tr}[x ; t]$ at $t=0$ implies that $\operatorname{Tr}[x ; t]$ is continuous in the variable $t$ for all $t$ and all $x \in \Omega_{1}$. Thus we need only to prove that $\operatorname{Tr}[x ; t]=x$ for rational $t$ 's and for a subset of full measure of $x$ 's in $\Omega_{1}$ : henceforth the reader may assume that $t$ is a rational number.

Let us consider a frame $f \in F M_{1}$ such that $f$ belongs to $\operatorname{pr}_{1}^{-1}\left(\Omega_{1}\right)$; for such an $f$ let us define $A_{t}(f) \in \mathbb{R}^{n-1}$ in such a way that $n_{\mathbf{A}_{t}(f)}(f)$ is the point on the horosphere of $f$ that projects in $T^{1} M_{1}$ to $\operatorname{Tr}\left[\mathrm{pr}_{1}(f) ; t\right]$.

Notice that if $x \in \Omega_{1}$ and $\tilde{x}$ is another point on the same horosphere of $x$, the horoline from $\tilde{x}$ to $\operatorname{Tr}[\tilde{x} ; t]$ will be parallel to and will have the same length as the horoline from $x$ to $\operatorname{Tr}[x ; t]$ (this is in fact a consequence of the proposition 3.1 and the fact that $\psi$ is an isometry on horospheres). Thus the map $f \mapsto \mathbb{A}_{t}(f)$ is a bounded measurable map which is constant along the horospheres of $F M_{1}$. By the ergodicity of the horospherical foliation of $F M_{1}$, we have that there exists a subset 
$\Omega_{t}$ of $\operatorname{pr}_{1}^{-1}\left(\Omega_{1}\right)$ entirely made of horospheres such that $\mathbb{A}_{t}(f)$ equals a constant $A_{t}$ for all $f \in \Omega_{t}$.

Let $K$ be a rotation in SO $(n-1)$ and $K(f)$ be the frame obtained by rotating $f$ by $K$; one has that

$$
\mathbb{A}_{t}(K(f))=\mathbb{A}_{t}(f) \cdot K
$$

$K(f)$ belongs to $\Omega_{t}$ for almost every $f \in \Omega_{t}$ and for almost every $K \in \operatorname{SO}(n-1)$, and therefore

$$
\mathbb{A}_{t}=\mathbb{A}_{t} \cdot K \quad \text { for almost every } K,
$$

which obviously implies $A_{t}=0$.

This is true for all rational $t$, hence there is a set of full measure of points $x$ in $\Omega_{1}$ such that $\operatorname{Tr}[x ; t]=x$ for all rational $t$ - and therefore for all real $t$. We have therefore proved the following proposition:

Proposition 3.2. Let $\psi: T^{1} M_{1} \mapsto T^{1} M_{2}$ be a bi-measurable isomorphism of the expanding horospherical foliation. Then $\psi$ coincides on a set of full measure with a map $\bar{\psi}: T^{1} M_{1} \mapsto T^{1} M_{2}$ with the property that

$$
g_{t}(\bar{\psi}(x))=\bar{\psi}\left(g_{t}(x)\right)
$$

for all $t$ and almost all $x \in T^{1} M_{1}$.

\section{Investigation of the effect of $\psi$ on contracting horospheres}

Thanks to proposition 3.2 we are now in a situation in which we can assume that we have a bi-measurable map

$$
\psi: \Omega \subset T^{1} M_{1} \rightarrow T^{1} M_{2},
$$

which is defined on a set $\Omega$ of measure 1 of weakly unstable leaves, with the property that:

(1) $\psi g_{t}(x)=g_{t} \psi(x), \quad \forall t \in \mathbb{R}, \forall x \in \Omega$;

(2) $\psi$ maps expanding horospheres to expanding horospheres isometrically.

To investigate further the properties of this map we need to define the notion of conjugacy of contracting and expanding horospheres.

Let $\left(u_{s}\right)_{s \in I}$ be a possibly infinite arc of expanding horoline and assume that $0 \in I$; then, if $f_{0}$ is a frame that projects down to $u_{0}$, the horoline $\left(u_{s}\right)_{s \in I}$ lifts to a horocycle $\left(n_{s \mathbf{a}} f_{0}\right)_{s \in I}$ for some $\left(a_{2}, \ldots, a_{n}\right) \in \mathbb{S}^{n-2}$, where $\mathbb{S}^{n-2}$ is the unit sphere in $\mathbb{R}^{n-1}$; the contracting horoline through $u_{0}$ conjugate to $\left(u_{s}\right)$ will be defined to be the projection to $T^{1} M_{1}$ of the contracting horocycle $\left.\left(m_{s \mathbf{a}} f_{0}\right)_{s \in \mathbb{R}}\right)$ with the same $\left(a_{2}, \ldots, a_{n}\right) \in \mathbb{S}^{n-2}$.

Notice that conjugate horolines through a point $u_{0}$ and the geodesic through $u_{0}$ lie in a three-dimensional manifold which can be thought of as the unit tangent bundle of a two-dimensional hyperbolic surface sitting in $M_{1}$.

The notion of conjugacy of horolines also introduces a correspondence among points of the expanding and contracting horospheres of a point $u_{0} \in T^{1} M_{1}$ : in fact if $v$ is a point on the expanding (contracting) horosphere of $u_{0}$ we can define its conjugate to be the point $\bar{v}$ on the contracting (expanding) horosphere of $u_{0}$ such that the horoline from $u_{0}$ to $v$ is conjugate to and has the same length as the horoline from $u_{0}$ to $\bar{v}$. 
As usual when we say 'almost every point on a horosphere' we will refer implicitiy to the euclidean measure on the horosphere.

Lemma 4.1. Assume that $\psi$ satisfies, besides the properties (1) and (2), the following property:

(3) there exists a set $\Omega_{1} \subset \Omega$ of measure 1 such that for all $u_{0} \in \Omega_{1}$ and almost every point $w$ on the contracting horosphere of $u_{0}$ one has that: (a) $w$ is mapped to a point which lies on the contracting horosphere of $\psi\left(u_{0}\right)$; (b) $\psi(w)$ is conjugate to the point $\psi(v), v$ being the point conjugate to $w$ on the expanding horosphere of $u_{0}$.

Then we can conclude that:

(a) $\exists \varepsilon>0$ such that $\forall u \in \Omega_{2}$, where $\Omega_{2}$ is defined by

$$
\Omega_{2} \stackrel{\operatorname{def}}{=}\left\{v \mid \begin{array}{c}
\text { the weakly unstable leaf of } v \\
\text { intersects } \Omega_{1} \text { on a set of full measure }
\end{array}\right\},
$$

one has that the restriction of $\psi$ to the ball of radius $\varepsilon$ around $u$ coincides almost everywhere with a continuous map.

This implies that:

(b) $\psi$ coincides a.e. on $\Omega$ with a continuous map $\tilde{\psi}: T^{1} M_{1} \rightarrow T^{1} M_{2}$ which satisfies the following properties:

(1) $\tilde{\psi}$ commutes with the geodesic flow;

(2) $\tilde{\psi}$ maps contracting as well as expanding horospheres to contracting and expanding horospheres isometrically;

(3) conjugate points on the horospheres of any point $u \in T^{1} M_{1}$ are mapped to conjugate points on the horospheres of $\tilde{\psi}(u)$.

Proof. By the compactness of $T^{1} M_{1}$ there exist constants $\varepsilon$ and $C$ such that, if two points $u, v \in T^{1} M_{1}$ have distance smaller than $\varepsilon$, then the disc of radius $C$ about $u$ in the weakly unstable leaf of $u$ intersects the disc of radius $C$ about $v$ in the contracting horosphere of $v$ in a unique point $[u, v]$.

Let $u_{0} \in \Omega_{2}$ and fix a system of rectangular coordinates on the expanding horosphere of $u_{0}$. Every point $w$ in the weakly unstable leaf of $u_{0}$ can be given coordinates $\left(t, w_{2}, \ldots, w_{n}\right) \in \mathbb{R}^{n}$, where $t$ is the number such that $g_{t}(w)$ belongs to the expanding horosphere of $u_{0}$ and $\left(w_{2}, \ldots, w_{n}\right)$ are the coordinates of $g_{t}(w)$ on such horosphere.

Then every point $z$ in an $\varepsilon$-ball around $u_{0}$ can be given coordinates

$$
\left(t, z_{2}, \ldots, z_{n}, \tilde{z}_{2}, \ldots, \tilde{z}_{n}\right)
$$

in the following way: $\left(t, z_{2}, \ldots, z_{n}\right)$ will be the coordinates of $\left[u_{0}, z\right]$ and $\left(\tilde{z}_{2}, \ldots, \tilde{z}_{n}\right)$ will be the coordinates of $z$ in the contracting horosphere of $\left[u_{0}, z\right]$ with respect to the rectangular system of coordinates conjugate to the system of coordinates on the horosphere of $u_{0}$.

In this way we have defined a (real analytic) one-to-one map from a neighbourhood of 0 in $\mathbb{R}^{2 n-1}$ onto the ball of radius $\varepsilon$ around $u_{0}$. The hypotheses (1) and (2) on the map $\psi$ imply that whenever $u_{0}$ belongs to $\Omega, \psi$ maps the rectangular system of coordinates on the expanding horosphere of $u_{0}$ to a rectangular system of coordinates on the expanding horosphere of $\psi\left(u_{0}\right)$ : points which have the given coordinates on 
the weakly unstable leaf of $u_{0}$ are mapped to points which have the same coordinates in the weakly unstable leaf of $u_{0}$.

The hypothesis (3) allows us to say that for all $u_{0} \in \Omega_{2}$ and for almost every $\left(t, u_{2}, \ldots, u_{n}, 0, \ldots, 0\right)$, the point with such coordinates in the $B_{\varepsilon}\left(u_{0}\right)$ belongs to $\Omega_{1}$ and therefore for almost every $\left(w_{2}, \ldots, w_{n}\right)$ the point of coordinates $\left(t, u_{2}, \ldots, u_{n}, w_{2}, \ldots, w_{n}\right)$ is mapped to the point with the same coordinates in $B_{\varepsilon}\left(\psi\left(u_{0}\right)\right)$. Thus $\psi$ coincides a.e. with the map which sends points in $B_{\varepsilon}\left(u_{0}\right)$ to points with the same coordinates in $B_{\varepsilon}\left(\psi\left(u_{0}\right)\right)$. This completes the proof of (a).

The proof of (b) is trivial because we can cover $T^{1} M_{1}$ with balls $B_{i}$ around points $u_{i}$ in $\Omega_{2}$ and in each of these balls $\psi$ coincides with a continuous map $\psi_{i}$ defined on $B_{i}$; but $\psi_{i}\left|B_{i} \cap B_{j}=\psi_{j}\right| B_{i} \cap B_{j}$ whenever $B_{i} \cap B_{j} \neq \varnothing$; so the collection of maps $\psi_{i}$ define a unique continuous function $\tilde{\psi}$ on $T^{1} M_{1}$ which satisfies the conditions (1), (2), (3) on a dense set of points and therefore at every point.

Now we will proceed to construct a set $\Omega_{1}$ for which condition (3) of the above lemma holds.

Let $\Lambda_{0}$ be a subset of $\Omega$ on which $\psi$ is uniformly continuous; $\Lambda_{0}$ can be chosen of measure larger than $1-\xi$ for every $\xi>0$; we assume $\xi<\frac{1}{6}$. Let $\tilde{\Lambda}_{0}$ be the pre-image of $\Lambda_{0}$ in $F M_{1}$ under the projection map

$$
F M_{1} \stackrel{\mathrm{pr}_{1}}{\longrightarrow} T^{1} M_{1}
$$

by the ergodicity of the horocycle flow $h_{t}(f)={ }^{\text {def }} n_{t(1,0, \ldots, 0)}(f)$ we have that $\exists L_{0}$ and $\tilde{\Lambda}_{1} \subset \operatorname{pr}_{1}^{-1}(\Omega)$ such that:

(a) the measure of $\tilde{\Lambda}_{1}$ is greater than $1-\xi$;

(b) $\forall f \in \tilde{\Lambda}_{1}, \forall \lambda>L_{0},(1 / \lambda)$ meas $\left.\{t \in \mid 0, \lambda] \mid h_{t}(f) \in \tilde{\Lambda}_{0}\right\}>1-2 \xi$.

Now let $\tilde{\Lambda}_{2}$ be the subset of $\operatorname{pr}_{1}^{-1}(\Omega)$ defined by the condition

$$
f \in \tilde{\Lambda}_{2} \Rightarrow \lim _{t \rightarrow \infty} \frac{1}{t} \int_{0}^{t} \chi \tilde{\Lambda}_{0} \cap \tilde{\Lambda}_{1}\left(g_{s} f\right) d s=\tilde{\mu}\left(\tilde{\Lambda}_{0} \cap \tilde{\Lambda}_{1}\right) \geq 1-2 \xi>\frac{2}{3} .
$$

The set $\tilde{\Lambda}_{2}$ by ergodicity has measure 1 in $F M_{1}$. Define

$$
\tilde{\Lambda}_{3} \stackrel{\text { def }}{=} \tilde{\Lambda}_{2} \cap\left\{f \in F M_{1} \mid m_{t(1,0, \ldots, 0)} f \in \tilde{\Lambda}_{2} \text { for almost every } t\right\}
$$

(notice that $m_{t(1,0, \ldots, 0)}$ is the contracting horocycle flow conjugate to $h_{t}$ ). Finally define

$$
\Omega_{1} \stackrel{\text { def }}{=} \Omega \cap\left\{v \in T^{1} M_{1} \mid \operatorname{pr}_{1}^{-1}(\{v\}) \cap \tilde{\Lambda}_{3} \text { is a set of full measure in } \operatorname{pr}_{1}^{-1}(\{v\})\right\} .
$$

We claim that, if $\xi$ is chosen sufficiently small, $\Omega_{1}$ is a set that enjoys the property (3) of lemma 4.1.

In fact if $v$ belongs to $\Omega_{1}$ then for almost every direction on the contracting horosphere of $v$, the contracting horoline through $v$ with that direction has the property that for almost every point $w$ on that horoline the following statements are true:

$(*)$ if $\left(v_{s}\right)_{s=0}^{\beta},\left(w_{s}\right)_{s=0}^{\beta}$ are arcs of expanding horolines through $v=v_{0}$ and $w=w_{0}$ which are conjugate to the contracting horoline from $v$ to $w$, then for infinitely many times $t$ (in fact with frequency at least $\frac{1}{3}$ ) one has that: 
(1) the images under the geodesic flow $g_{t}$ of the $\operatorname{arcs}\left(v_{s}\right)_{s=0}^{\beta}$ and $\left(w_{s}\right)_{s=0}^{\beta}$ are arcs of expanding horolines, of length greater than $L_{0}$, which visit the set $\Lambda_{0}$, on which $\psi$ is uniformly continuous, with frequency greater than $1-2 \xi$;

(2) $g_{t}\left(v_{0}\right)$ and $g_{t}\left(w_{0}\right)$ belong to the continuity set $\Lambda_{0}$.

Note also that, by our definition, $v$ and $w$ belong to $\Omega$ and therefore $\psi$ maps their weakly unstable leaves 'nicely' in $T^{1} M_{2}$.

Proposition 4.2. If $v$ and $w$ are points satisfying the conditions of paragraph $(*)$ we can say that:

(a) $\psi(v)$ and $\psi(w)$ are points in $T^{1} M_{2}$ belonging to the same contracting horosphere; and moreover

(b) the arcs of expanding horolines $\left(v_{s}\right)_{s=0}^{\beta},\left(w_{s}\right)_{s=0}^{\beta}$ are mapped exactly to the arcs of expanding horolines through $\psi(v)$ and $\psi(w)$ which are conjugate to the contracting horoline from $\psi(v)$ to $\psi(w)$.

(c) the length of the contracting horoline from $v$ to $w$ equals the length of the contracting horoline from $\psi(v)$ to $\psi(w)$.

Now it is easy to understand that the conclusions of the above proposition together with the facts established before, are merely a more symmetric rephrasing of the condition (3) of lemma 4.1; in fact we have seen that for all $v \in \Omega_{1}$ (and $\Omega_{1}$ is a set of measure (1) and for almost every point $w$ on the contracting horosphere of $v$ the conditions of paragraph $(*)$ are true; then proposition 4.2 says that, by $(a), \psi(w)$ lies on the horosphere of $\psi(v)$, and, by (b), $\psi(w)$ is conjugate to the point $\psi\left(v_{\gamma}\right)$ on the horoline $\left(\psi\left(v_{s}\right)\right)_{s \in \mathbb{R}}$ that extends the horoline $\left(\psi\left(v_{s}\right)\right)_{s=0}^{\beta}$. But, by $(\mathrm{c}), w$ is conjugate to $v_{y}$ and therefore we have that $\psi(w)$ is conjugate to the image under $\psi$ of the point to which $w$ is conjugate. So, if the above proposition is true, we see that $\Omega_{1}$ is a subset of $\Omega$ that satisfies the condition (3) of lemma 4.1.

We shall postpone the proof of the above proposition to next section; for now let us notice that from proposition 3.2, proposition 4.2 and lemma 4.1 we have the following

Proposition 4.3. Let $\psi: T^{1} M_{1} \rightarrow T^{1} M_{2}$ be a measurable map which maps expanding horospheres to expanding horospheres isometrically. Then $\psi$ coincides a.e. with a continuous map $\tilde{\psi}: T^{1} M_{1} \rightarrow T^{1} M_{2}$ which commutes with the geodesic flow, maps expanding and contracting horospheres isometrically to expanding and contracting horospheres and sends conjugate horolines to conjugate horolines.

We can now prove the following theorem:

THEOREM 1. Let $M_{i}={ }^{\text {def }} \Gamma_{i} \backslash \mathrm{SO}_{0}(1, n) / \mathrm{SO}(n)$ and let $\psi: T^{1} M_{1} \rightarrow T^{1} M_{2}$ be a measurable map which sends expanding horospheres to expanding horospheres isometrically; then $\psi=d \phi$ almost everywhere, $\phi$ being an isometry from $M_{1}$ to $M_{2}$.

Proof. We claim that a continuous map $\tilde{\psi}: T^{1} M_{1} \rightarrow T^{1} M_{2}$ satisfying the conclusion of proposition 4.3 is the lift to the unit tangent bundles of an isometry $\phi: M_{1} \rightarrow M_{2}$. The proof of this claim is trivial: we want to prove that if $u, v$ are vectors in $T^{1} M_{1}$ at the same point $p \in M_{1}$, then $\psi(u)$ and $\psi(v)$ are vectors at the same point in $M_{2}$; 
if this is the case then there is a unique map $\phi: M_{1} \rightarrow M_{2}$ such that the diagram

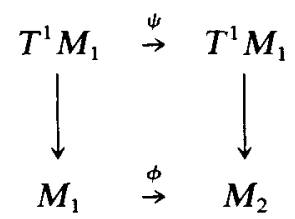

commutes; $\phi$ will be an isometry because if $p_{1}, p_{2}$ belong to $M_{1}$ and $\gamma\left(p_{1}, p_{2}\right)$ is the shortest geodesic connecting $p_{1}$ to $p_{2}$, by lifting the geodesic to $T^{1} M_{1}$, mapping over in $T^{1} M_{2}$ and projecting down in $M_{2}$, we see that $\phi\left(p_{1}\right)$ and $\phi\left(p_{2}\right)$ have the same distance as $p_{1}$ and $p_{2}$.

To prove the claim that vectors above the same point in $M_{1}$ are mapped to vectors above the same point in $M_{2}$ it is enough to prove it locally. Assume that $u, v$ are vectors in $T^{1} M_{1}$ that project to the same point $p \in M_{1}$ and $d(u, v)<\delta$; let $U$ be a disc of radius $\varepsilon$ in the surface containing $p$, tangent to $u$ and $v$ and locally isometric to a subset of $\mathbb{O}^{2}$; then $u, v$ can be uniquely connected by a path in $T^{1} U \subset T^{1} M_{1}$ made of three arcs $\gamma_{1}, \gamma_{2}, \gamma_{3}$, where

(a) $\gamma_{1}$ is an arc of expanding horoline from $u=u_{0}$ to $u_{1}$;

(b) $\gamma_{2}$ is a (conjugate) arc of contracting horoline from $u_{1}$ to $u_{2}$;

(c) $\gamma_{3}$ is an arc of geodesic from $u_{2}$ to $u_{3}=v$.

The corresponding arcs in $T^{1} M_{2}$ to which $\psi$ maps $\gamma_{1}, \gamma_{2}, \gamma_{3}$ will also lie in the tangent bundle to some two-dimensional surface locally isometric to hyperbolic two-space $H^{2}$ and will have the same lengths; therefore their projections in $M_{2}$ will give a path which closes up in $M_{2}$; and this completes the proof of our claim.

The above theorem can be rephrased in the following way:

THEOREM 1'. Under the hypotheses of theorem 1, one can conclude that there exists $g \in O(1, n)$ such that

$$
\Gamma_{2}=g \Gamma_{1} g^{-1}
$$

and the map $\phi$ is given by

$$
\phi\left(\Gamma_{1} x\right)=\Gamma_{2} g x=g \Gamma_{1} x .
$$

Proof. An isometry $\phi: M_{1} \rightarrow M_{2}$ induces an isometry of the universal covers of $M_{1}$ and $M_{2}$ and such an isometry is given by a map

$$
x \in \mathbb{H}^{n} \mapsto g x \in \mathbb{H}^{n},
$$

where $g \in O(1, n)$. But the diagram

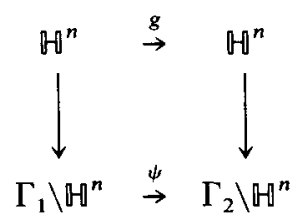

commutes if and only if $\Gamma_{2}=g \Gamma_{1} g^{-1}$.

Now we turn to the proof of proposition 4.2. 


\section{Proof of proposition 4.2}

The ideas of the proof of proposition 4.2 follow the lines of [Ra]; we need to estimate the distance between starting points of horolines which stay close for time $L$.

LEMMA 5.1. Let $\left(u_{s}\right),\left(v_{s}\right)$ be horolines in $T^{1} \mathbb{H}^{n}$ such that $d\left(u_{s}, v_{s}\right)<\varepsilon$ for all $0 \leq s \leq L$ and $\varepsilon$ sufficiently small; then $v_{0}$ can be connected to $u_{0}$ by a path made of three arcs $\gamma_{1}, \gamma_{2}, \gamma_{3}$, where:

(a) $\gamma_{1}$ is an arc of expanding horoline of length less than $C \varepsilon$;

(b) $\gamma_{2}$ is an arc of contracting horoline of length less than $C \in L^{-2}$;

(c) $\gamma_{3}$ is an arc of geodesic of length less than $C \varepsilon$.

Proof. By the left invariance of the metric $d$ we can assume that $u_{0}$ is the vector $\epsilon$ of coordinates

$$
\boldsymbol{\epsilon}=\left(\begin{array}{cc}
1 & 0 \\
0 & 1 \\
0 & 0 \\
\vdots & \vdots \\
0 & 0
\end{array}\right)
$$

and $v_{0}$ is some vector such that $d\left(\epsilon, v_{0}\right)<\varepsilon$. Then by the transversality of the horospherical foliations and the geodesic flow we can find a unique path $\gamma_{1}, \gamma_{2}, \gamma_{3}$ of arcs as in the statement of the lemma such that each of these arcs has length less than $C \varepsilon$, where $C$ is some positive constant. Let $f_{0}$ be the frame

$$
\left(\begin{array}{cccc}
1 & 0 & \cdots & 0 \\
0 & 1 & \cdots & 0 \\
\vdots & & \ddots & \\
0 & 0 & \cdots & 1
\end{array}\right)
$$

then a frame covering $v_{0}$ is given by

$$
n_{\alpha \mathbf{a}} m_{\beta \mathbf{b}} G_{\gamma}
$$

where $|\alpha|<C \varepsilon,|\beta|<C \varepsilon,|\gamma|<C \varepsilon$. Horolines of length $L$ from $\epsilon$ and $v_{0}$ will be given by the projection in $T^{1} \mathbb{H}^{n}$ of the orbits

$$
\left(n_{s \mathbf{c}}\right)_{s=0}^{L} \quad\left(\left(n_{\alpha \mathbf{a}} m_{\beta \mathbf{b}} G_{\gamma}\right) n_{s \mathbf{d}}\right)_{s=0}^{L} .
$$

By the left invariance under $\mathrm{SO}_{0}(1, n)$ of metric $d$ on $T^{1} \mathbb{H}^{n}$ we have:

$$
d\left(\operatorname{pr}_{1}\left(n_{s \mathrm{c}}\right), \mathrm{pr}_{1}\left(\left(n_{\alpha \mathrm{a}} m_{\beta \mathrm{b}} G_{\gamma}\right) n_{s \mathrm{~d}}\right)\right)=d\left(\epsilon, \mathrm{pr}_{1}\left(n_{-s \mathrm{c}} n_{\alpha \mathrm{a}} m_{\beta \mathrm{b}} G_{\gamma} n_{s \mathrm{~d}}\right)\right) ;
$$

after tedious computations one can show that the entries of the first two columns of the matrix

$$
n_{-s \mathrm{c}}\left(n_{\alpha \mathbf{a}} m_{\beta \mathbf{b}} G_{\gamma}\right) n_{s \mathrm{~d}}
$$

are polynomials in $s$ whose common leading term is given by

$$
2 e^{-\gamma} \beta^{2} s^{4}
$$

Since such entries must be less than a constant times $\varepsilon$ for all $0 \leq s \leq L$ one has that

$$
\beta<\text { Const } \varepsilon / L^{2} \text {. }
$$

And this concludes the proof of lemma 5.1. 
For the convenience of the reader, let us now restate proposition 4.2.

Proposition 4.2. Let $v_{0}$ and $w_{0}$ be points in $\Omega$ that belong to the same horosphere and let $\left(v_{s}\right)_{s=0}^{\beta_{1}}$ and $\left(w_{s}\right)_{s=0}^{\beta_{2}}$ be arcs of expanding horolines through $v_{0}$ and $w_{0}$ which are conjugate to the contracting horoline from $v_{0}$ to $w_{0}$. Assume that there are infinitely many t's such that:

(1) the images under the map $g_{t}$ of the arcs $\left(v_{s}\right)_{s=0}^{\beta_{1}}$ and $\left(w_{s}\right)_{s=0}^{\beta_{2}}$ are arcs of expanding horolines, of length greater than $L_{0}$, which visit the set $\Lambda_{0}$ (on which $\psi$ is uniformly continuous) with frequency greater than $1-2 \xi$;

(2) the points $g_{t} v_{0}$ and $g_{t} w_{0}$ belong to the continuity set $\Lambda_{0}$.

Then we can say that:

(a) $\psi\left(v_{0}\right)$ and $\psi\left(w_{0}\right)$ are points in $T^{1} M_{2}$ belonging to the same contracting horosphere; and moreover

(b) the arcs of expanding horolines $\left(v_{s}\right)_{s=0}^{\beta},\left(w_{s}\right)_{s=0}^{\beta}$ are mapped exactly to the arcs of expanding horolines through $\psi\left(v_{0}\right)$ and $\psi\left(w_{0}\right)$ which are conjugate to the contracting horoline from $\psi\left(v_{0}\right)$ to $\psi\left(w_{0}\right)$;

(c) the length of the contracting horoline from $v_{0}$ to $w_{0}$ equals the length of the contracting horoline from $\psi\left(v_{0}\right)$ to $\psi\left(w_{0}\right)$.

Proof. Without loss of generality we can assume that the distance between $v_{0}$ and $w_{0}$ is as small as we like. Let $w_{q(s)}$ be the point where the weakly stable leaf through $v_{s}$ intersects the horoline $\left(w_{s}\right) ; q(s)$ is a function which depends only upon $s$ and the distance between $v_{0}$ and $w_{0}$, since the horolines $\left(v_{s}\right)$ and $\left(w_{s}\right)$ are conjugate to the same contracting horoline. Moreover we have

$$
\begin{array}{ll}
\sup _{0 \leq s \leq 1}|q(s)-s| \rightarrow 0 & \text { as } d\left(v_{0}, v_{0}\right) \rightarrow 0 \\
\sup _{0 \leq s \leq 1}\left|\frac{d q}{d s}-1\right| \rightarrow 0 & \text { as } d\left(v_{0}, v_{0}\right) \rightarrow 0 .
\end{array}
$$

Fix an $\varepsilon>0$; corresponding to this $\varepsilon$ there is a $\eta$ such that

$$
d(x, y)<\eta \text { and } x, y \in \Lambda_{0} \Rightarrow d(\psi(x), \psi(y))<\varepsilon .
$$

Let the distance between $v_{0}$ and $w_{0}$ be so small that for all $t>0$

(a) $\sup _{0 \leq s \leq 1} d\left(g_{t}\left(v_{s}\right), g_{t}\left(w_{q(s)}\right)\right)<\eta$;

(b) $\sup _{0 \leq s \leq 1}|q(s)-s|<\alpha$;

(c) $\sup _{0 \leq s \leq 1}|(d q / d s)-1|<\alpha$, where $\alpha=\alpha(\xi)$ is a number to be defined later.

Then the horolines

$$
\left(g_{t} v_{s}\right)_{s=0}^{1} \quad\left(g_{t} w_{s}\right)_{s=0}^{q(1)}
$$

have lengths greater than $e^{t} \min (1, q(1))>e^{t}(1-\alpha)$ which for large $t$ will be greater than $L_{0}$. By our assumptions there are infinitely many $t$ for which these horolines will visit the continuity set $\Lambda_{0}$ with frequency greater than $1-2 \xi$. This implies

$$
\int_{0}^{1} \chi_{\Lambda_{0}}\left(g_{i} v_{s}\right) d s>1-2 \xi \text { and } \int_{0}^{1} \chi_{\Lambda_{0}}\left(g_{t} w_{\tau}\right) d \tau>1-2 \xi
$$


But then

$$
\begin{aligned}
\int_{0}^{1} \chi_{\Lambda_{0}}\left(g_{t} w_{q(\sigma)}\right) d \sigma & \geq \frac{1}{1+\alpha} \int_{0}^{1} \chi_{\Lambda_{0}}\left(g_{t} w_{q(\sigma)}\right) \frac{d q}{d s} d \sigma \\
& =\frac{1}{1+\alpha} \int_{0}^{q(1)} \chi_{\Lambda_{0}}\left(g_{t} w_{\tau}\right) d \tau \\
& =\frac{1}{1+\alpha}\left(\int_{0}^{1}-\int_{q(1)}^{1}\right) \\
& \geq \frac{1}{1+\alpha}(1-2 \xi-\alpha) .
\end{aligned}
$$

So if we choose $\alpha$ small enough we have

$$
\int_{0}^{1} \chi_{\Lambda_{0}}\left(g_{t} w_{q(s)}\right) d s>1-3 \xi
$$

and therefore for at least a fraction $(1-5 \xi)$ of $s$ 's one has simultaneously

$$
g_{t} v_{s} \in \Lambda_{0} \quad g_{t} w_{q(s)} \in \Lambda_{0} .
$$

Therefore for these s's, since $d\left(g_{t}\left(v_{s}\right), g_{t}\left(w_{q(s)}\right)\right)<\eta$, one has

$$
d\left(\psi\left(g_{t}\left(v_{s}\right)\right), \psi\left(g_{t}\left(w_{q(s)}\right)\right)\right)=d\left(g_{t}\left(\psi\left(v_{s}\right)\right), g_{t}\left(\psi\left(w_{q(s)}\right)\right)\right)<\varepsilon .
$$

Let $\bar{w}_{0}$ be the point on the contracting horoline of $\psi\left(v_{0}\right)$ conjugate to the horoline $\psi\left(v_{s}\right)_{s=0}^{1}$, whose distance from $\psi\left(v_{0}\right)$ equals the distance of $w_{0}$ from $v_{0}$. Let $\left(\bar{w}_{s}\right)$ be the expanding horoline through $\bar{w}_{0}$ conjugate to the horoline from $\psi\left(v_{0}\right)$ to $\bar{w}_{0}$. We have

$$
d\left(g_{t} \bar{w}_{q(s)}, g_{t} \psi\left(v_{s}\right)\right)<\eta
$$

for all $t>0$ and $0 \leq s \leq 1$; therefore

$$
d\left(g_{t} \bar{w}_{q(s)}, g_{t}\left(\psi\left(w_{q(s)}\right)\right)\right)<\varepsilon+\eta,
$$

whenever $g_{t} v_{s}$ and $g_{t} w_{q(s)}$ belong to $\Lambda_{0}$. But then

$$
\begin{aligned}
\int_{0}^{q(1)} d\left(g_{t} \bar{w}_{\sigma}, g_{t}\left(\psi\left(w_{\sigma}\right)\right)\right) d \sigma & =\int_{0}^{1} d\left(g_{t} \bar{w}_{q(\sigma)}, g_{t}\left(\psi\left(w_{q(\sigma)}\right)\right)\right) \frac{d q}{d s} d \sigma \\
& <(1+\alpha) \int_{0}^{1} d\left(g_{t} \bar{w}_{q(\sigma)}, g_{t}\left(\psi\left(w_{q(\sigma)}\right)\right)\right) d \sigma \\
& <(1+\alpha)(\varepsilon+\eta+5 \xi D),
\end{aligned}
$$

where $D$ is the radius of injectivity of $T^{1} M_{2}$. By lemma 2.1 if $\xi$ and $\alpha$ are chosen small enough, this implies that

$$
d\left(g_{t} \bar{w}_{s}, g_{t}\left(\psi\left(w_{s}\right)\right)\right)<C \varepsilon \quad \text { for all } 0 \leq s \leq q(1) ;
$$

the horolines $\left(g_{t} \bar{w}_{s}\right)_{s=0}^{q(1)}$ and $\left(g_{t}\left(\psi\left(w_{s}\right)\right)\right)_{s=0}^{q(1)}$ have length greater than $e^{t} q(1)>1 / 2 e^{t}$ and therefore by the lemma 5.1 we have that $g_{t} \bar{w}_{0}$ and $g_{t}\left(\psi\left(w_{0}\right)\right)$ can be connected by a path $\gamma_{1}, \gamma_{2}, \gamma_{3}$ where $\gamma_{1}$ and $\gamma_{3}$ are arcs respectively of expanding horoline and of geodesic both of length less than $C_{1} \varepsilon$, and $\gamma_{2}$ is an arc of contracting horoline of length less than $C_{1} \varepsilon / e^{-2 t}$. This implies that $\bar{w}_{0}$ and $\psi\left(w_{0}\right)$ can be connected by 
a path $\gamma_{1}^{\prime}, \gamma_{2}^{\prime}, \gamma_{3}^{\prime}$, where:

(1) $\gamma_{1}^{\prime}$ is an arc of expanding horoline of length less than $C_{1} \varepsilon / e^{-t}$;

(2) $\gamma_{2}^{\prime}$ is an arc of contracting horoline of length less than $C_{1} \varepsilon / e^{-t}$;

(3) $\gamma_{3}^{\prime}$ is an arc of geodesic length less than $C_{1} \varepsilon$.

Since this holds for infinitely many positive values of $t$ we must have $\gamma_{1}^{\prime}=0, \gamma_{2}^{\prime}=0$ and $\bar{w}_{0}$ and $\psi\left(w_{0}\right)$ must lie on the same geodesic. We claim $\bar{w}_{0}=\psi\left(w_{0}\right)$; in fact, by the assumption (2), for some subsequence of $t$ 's we have that:

$$
d\left(g_{t} v_{0}, g_{t} w_{0}\right) \rightarrow 0 \Rightarrow d\left(\psi\left(g_{t} v_{0}\right), \psi\left(g_{t} w_{0}\right)\right) \rightarrow 0 ;
$$

on the other hand, since $\bar{w}_{0}$ and $\psi\left(w_{0}\right)$ are on the same geodesic, $d\left(\bar{w}_{0}, \psi\left(w_{0}\right)\right)=$ $d\left(g_{t} \bar{w}_{0}, g_{t} \psi\left(w_{0}\right)\right)$ for all $t$ and

$$
d\left(g_{t} \bar{w}_{0}, g_{t} \psi\left(w_{0}\right)\right) \leq d\left(g_{t} \bar{w}_{0}, g_{t} \psi\left(v_{0}\right)\right)+d\left(g_{t} \psi\left(v_{0}\right), g_{t} \psi\left(w_{0}\right)\right) \rightarrow 0
$$

therefore $\bar{w}_{0}=\psi\left(w_{0}\right)$. And this concludes the proof.

Added in proof. After this work was completed we have learned that D. Witte [Wi] has proved results similar to ours concerning the rigidity of the action of horospherical elements. More exactly his results imply the rigidity of the horospherical foliation of the frame bundle: let $\psi: \Gamma_{1} \backslash \mathrm{SO}_{0}(1, n) \rightarrow \Gamma_{2} \backslash \mathrm{SO}_{0}(1, n)$ be a measure preserving map that is an isometry when restricted to expanding horospheres. For each $x \in \Gamma_{1} \backslash S O_{0}(1, n)$ there is a $\phi_{x} \in \mathrm{SO}(n-1)$ with $\psi\left(n_{s \mathbf{a}} x\right)=n_{\phi_{x}(s \mathbf{s})} \psi(x)$; since $\phi_{x}=\phi_{y}$ whenever $x$ and $y$ are on the same horosphere $\phi_{x}$ is independent of $x$. In Witte's terminology, this means that $\psi$ is affine for the horospherical group. Hence Witte's theorem implies that $\psi$ is an affine map.

Acknowledgments. I am very thankful to my adviser Don Ornstein for his help and encouragement. I also would like to thank S. Kerkchoff, Y. Katznelson and J. King for useful conversations, and the A. P. Sloan Foundation for financial support during the last year of my graduate studies.

\section{REFERENCES}

[Ko-No] Kobayashi \& Nomizu. Foundation of Differential Geometry. Interscience Publishers, 1963.

[Mo] C. C. Moore. Ergodicity of flows on homogeneous spaces. Amer. J. Math. 88 (1966), 154-177.

[Ra] M. Ratner. Rigidity of the horocycle flow. Ann. of Math. 115 (1982), 597-614.

[Wi] D. Witte. Rigidity of some translations on homogeneous spaces. Invent. Math. 81 (1985), 1-27. 